\title{
Extending the Use of Full-Scale Lean Production Simulators: Their Role as Innovation Testbeds
}

\author{
Leo J DE VIN ${ }^{1}$, Emma JUNGHAGE, JanErik ODHE, and Lasse JACOBSSON \\ Karlstad University, Sweden
}

\begin{abstract}
This paper describes the use of a flexible full-scale simulation environment for Lean Production training and education called "KLF Karlstad Lean Factory ${ }^{\mathbb{B},}$. Instead of using the PDCA cycle as model for improvement cycles, the authors have developed a model that is more descriptive; it supports training transfer to the work environment in a more intuitive way. Recently, the authors have started to use the simulator as a testbed for innovative production solutions. Together with a company, the simulator is configured so as to emulate their envisaged future production solution. This participatory modelling \& simulation process consists of three main stages: (i) creating a common view on aim and scope, (ii) configuration modelling, and (iii) simulations. After the simulations, participants tend to continue seeking improvements, which illustrates the effectiveness of the approach. Future work will include developing a model for measuring lean production maturity in SMEs.
\end{abstract}

Keywords. Lean Production, Engineering Education, Training Within Industry, Participatory Modelling \& Simulation, Innovation Testbeds.

\section{Introduction}

The aim of this paper is to present and share some experiences from using a full-scale Lean Production (LP) simulator called Karlstad Lean factory ${ }^{\circledR}$ (KLF) as an innovation testbed. The paper focuses on small to medium-sized companies (SMEs) in the manufacturing sector. The difference between LP training and education on the one hand and using an LP simulator like KLF as testbed for innovative production solutions is that in the first case, the purpose of the training is for the participants to gain knowledge and experience of LP in general and to learn to adopt an "LP mind-set". In the latter case, the main purpose is to explore and study different production solutions for a specific scenario.

However, these two different uses of an LP simulator have one thing in common and that is transfer from training/testing in the simulator environment to the actual workplace. Training transfer is described by Luttik [1] as: "That almost magical link between classroom performance and something which is supposed to happen in real world". Something similar goes for using an LP simulator as innovation testbed: The proof of the pudding is in the eating and can be formulated as a question "To which

\footnotetext{
${ }^{1}$ Corresponding Author. leo.devin@kau.se
} 
degree do the experiences from the simulations result in an innovative production solution and in a culture of continually seeking improvement potential?".

What one essentially does in simulation-based LP training or in exploring alternative solutions for innovative production in an LP simulator is something called "experiential learning", described in more detail in [2]. Participants learn by doing but they also do from learning. However, models for experiential learning (e.g., Koops \& Hoevenaar, [3]) tend to be complex and may be suitable for use within experiential learning research, but they are of limited support to LP education practitioners and even less so for LP training participants. Likewise, models for continuous improvement such as PDCA (Plan-Do-Check-Act) have their limitations when training LP novices.

The disposition of the remainder of this paper is as follows: First, the limitations and other issues regarding the popular PDCA model are discussed, followed by the presentation of a dual model that is used by the authors to describe simulator-based training on the one hand and continuous improvement on the other hand. Next, the simulator KLF is described. Finally, the use of KLF as innovation testbed is described, including an overview of a typical scenario and associated model for interacting with a company.

\section{Models for learning and for continuous improvement}

Many models for game-based learning and for continuous improvement are experienced as complex and abstract, both by LP training participants and by LP educators. Models such as the Lemniscate model for experiential learning [3] may be suitable for educational research but they are usually too complex to support game scenario design and they are definitely too complex to explain the improvement and learning process to LP training participants. Furthermore, these models usually address neither training transfer to the work environment nor organisational learning.

A popular model to describe continuous improvement processes is the PDCA cycle (Plan-Do-Check-Act) shown in Figure 1, usually attributed to W. Edwards Deming. However, various interpretations of this cycle exist. Some authors replace "Act" with "Adjust", and others replace "Check" with "Learn" or "Study". The latter modification was according to Moen [4] also Deming's preferred interpretation as "Study" suggests an analysis and learning step which "Check" does not.
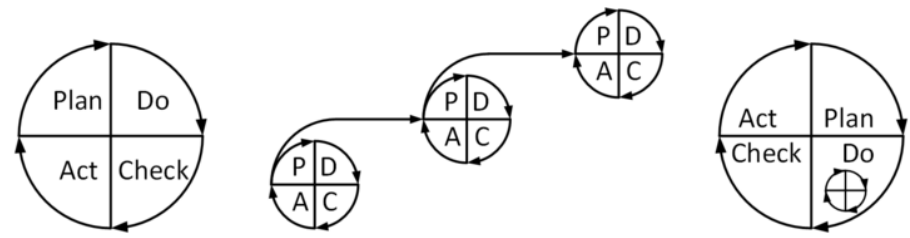

Fig. 1. Various alternative versions of the PDCA cycle

Moreover, various LP practitioners and consultants tend to use the PDCA model for completely different levels; from anything like small improvement steps in the work place to strategic decision making on business level [5]. This means that the descriptions of the steps and activities within each phase can differ quite much, 
depending on the organisational level and the type of the improvement process at hand. This often leads to confusion, according to Radziwill [6] even amongst professionals. Thus, despite its popularity, the PDCA model has drawbacks when explaining improvement processes to LP novices. Machine operators tend to experience it as abstract and stifling.

Having identified the difficulties that many LP training participants have in relating the training activities to the PDCA model, the authors developed a more descriptive model [2]. The model has more focus on how participants learn to see opportunities for improvement than on suggesting a problem-solving recipe. Responses from KLF training participants, students and industrial workers alike, are positive and indicate that the model is perceived as intuitive. With only some relatively minor changes, the same model can describe continuous improvement in the work environment. This dual model is shown in Figure 2. The left part describes simulatorassisted training, and the right part describes improvement cycles in the work environment in an almost identical way.
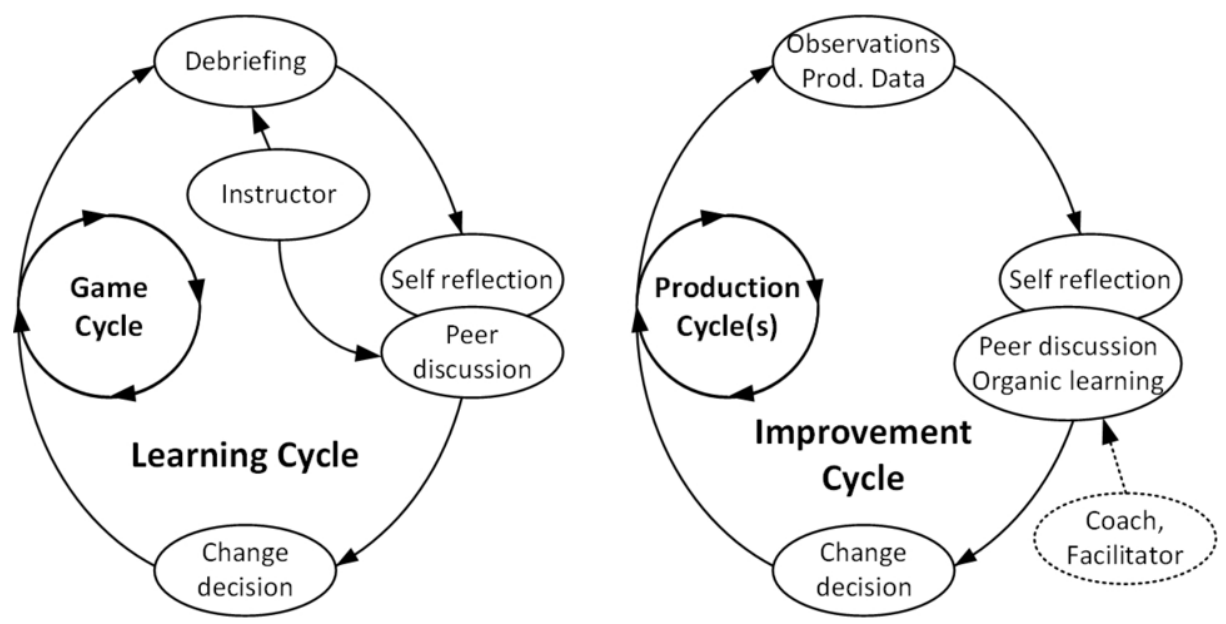

Fig. 2. Dual model for training (left) and continuous improvement at the workplace (right)

The advantage of this dual model is that not only is it an easy to grasp descriptive model, it also shows how similar the training activities are to improvement cycles in the work environment. There is experience-based evidence indicating that this stimulates training transfer. As an example, a few days after one training session with a small company, a group of blue-collar workers suggested to make an opening in a wall to reduce transports and to improve communication between departments. Asked by company management how they arrived at that idea they responded: "We started to look at the factory in the same way that we looked at KLF during training".

The dual model also illustrates the changing role of the instructor/facilitator as present inside the loop of the training cycle. In the improvement cycle, this instructor replaced by a coach/facilitator (outside the loop) whose role gradually decreases as organisational learning takes over. This difference over time illustrates a process called "instructional scaffolding" by Wood et al. [7]. During the training, the participants 
receive feedback mainly from the instructor but sometimes also from more experienced peers. This provides a "scaffolding" that allows the participants to reach a level of proficiency that they would not be able to reach on their own. At their workplace, this scaffolding is removed gradually: The coach or facilitator moves to the background while the participants individually and as a group make learning progress. It is not only possible for the coach to move to the background, it is also a condition for organisational learning. Learning takes place in what Dixon [8] calls "hallways of learning" where new collective meaning is constructed by a group through internal dialogue. Expert knowledge from the outside (such as from a coach) may inform this process, but cannot replace it. Too much intervention from the coach may actually hinder the exploration of innovative directions by the group.

\section{Karlstad Lean Factory ${ }^{\circledR}$ equipment}

The idea to design and build a new type of simulator emerged from discussions with manufacturing companies in the Värmland region of Sweden, many of them SMEs. They expressed that desktop games lacked realism for training production workers, and that simulators based on assembly alone had their limitations as well. This resulted in the design of KLF, described in more detail in [2]. The equipment represents single unit processing stations, batch processing stations, and assembly areas. It is flexible w.r.t. the type of product, but as standard product for training, the participants build children's playing chairs. The chairs are available in two colours, which makes it possible to simulate several production situations, including special orders through colour combinations. Apart from cycle times, average disturbance intervals (MTBF) and repair times (MTTR) can be set on each station. MTBF and MTTR are set for game scenarios in which disturbances and maintenance play a role. The stations will then generate random disturbances, which participants experience as more natural than when those are "generated" by a time-keeper or instructor. The equipment is mobile to facilitate reconfiguration during a training session and to enable onsite training. Figure 3 shows a group of students using KLF in a LP exercise at the university.

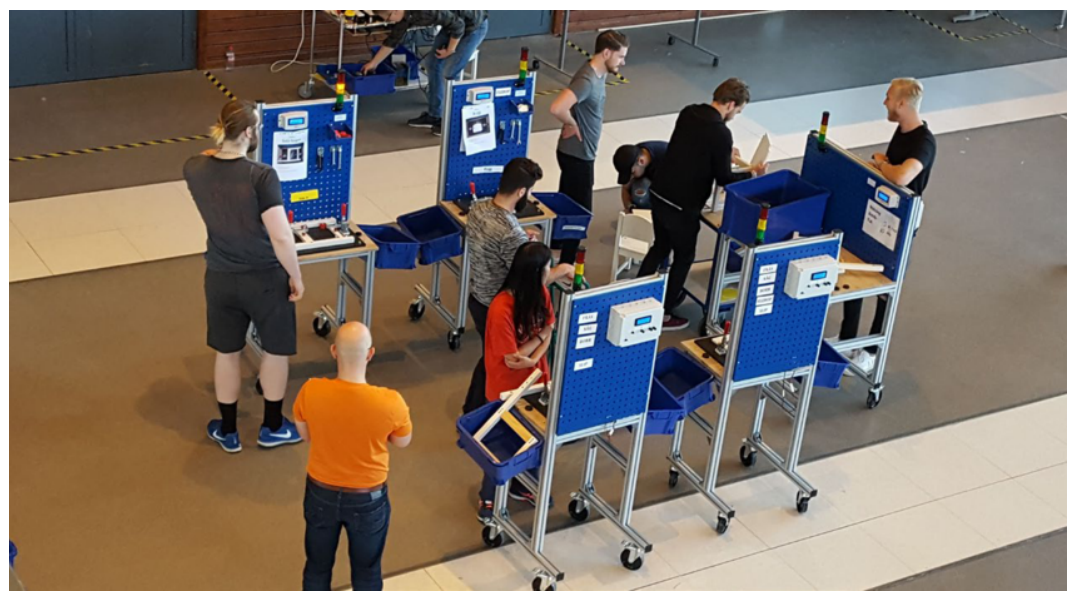

Fig. 3. Students training during a basic LP exercise using KLF 


\section{Using Karlstad Lean Factory ${ }^{\circledR}$ as innovation testbed}

Compared to other simulator types, full-scale LP simulators provide a very good mix of realism and flexibility [9]. In KLF, it is possible to simulate a range of environments, production environments in particular. This suggests that the simulator can be used as a testbed for a range of innovative production solutions, as discussed below.

\subsection{Participatory modelling and simulation}

Modelling an innovative future production solution in KLF can be challenging. However, this is an essential characteristic of using a model [10]. Moreover, it forces those who participate in the modelling activity to focus on the main characteristics of the envisaged production solution. Companies may wish to simulate with a product resembling their own products, but using a different product (such as the chair) has some advantages. AUDI AG for instance built an LP simulator described in [11] that is deliberately different from the press shop it simulates, so as not to stifle creativity. The simulator described in [11] is a sandwich bar for customised sandwiches. This not only plays down the formal and informal roles as they may exist in the press shop, it also changes the gender bias. Simulation of an envisaged production solution with physical equipment like KLF is immersive, which stimulates engagement and feedback from production operators. Once the overall design of a future production facility has been established, additional computer simulations can be used during an optimisation phase

Participatory modelling and simulation stimulates dialogue. This makes it easier to grasp tacit knowledge from shop floor operators, as it makes it easier for operators to give feedback on suggestions and to make own suggestions. Not only does it stimulate engagement, it also increases acceptance of the simulation results as the participants have been actively involved in (and could influence) all the steps leading to those results.

Stirna et al. [12] suggest five roles for participatory modelling: process owner, facilitator, modelling expert, modelling tool operator, and domain expert. When using physical simulators, some of these roles can be combined and one is superfluous. The process owner can be the production manager of a company (in the case of SMEs possibly the managing director). The facilitator typically is both a LP expert and an expert on the simulator (modelling expert). Thirdly, domain experts can be a variety of company staff, machine operators and assembly workers in particular. As physical equipment is used, there is no need for a programmer (modelling tool operator). A particular task for the facilitator is to monitor inclusion throughout the process. The authors see inclusion as an important indicator for LP maturity, but it is difficult to measure, in particular in SMEs (where it can be more difficult to raise specific cases of poor inclusion). By paying explicit attention to the importance of inclusion during the participatory modelling and simulation process, the company (or companies in case of a simulation involving suppliers and/or customers) is stimulated to pay continued attention to this aspect after the simulations. One way to bring attention for inclusion to the foreground is to assign various roles (such as non-native speaker, shy operator, sceptic operator) to the participants during the simulation and improvement discussions. This forces them to think and act differently than in their traditionally established roles that can be deeply coded into a group. 


\subsection{Experiences from using Karlstad Lean Factory ${ }^{\circledR}$ as an innovation testbed}

A KLF participatory modelling process usually starts with an initial discussion with management and some white-collar and blue-collar workers in order to define the objectives, scope and limitations of a simulation, followed by modelling involving staff representing all internal stakeholders in the new production solution. Ideally, all production-related staff is involved, but this is not always practically possible for larger SMEs. In one case described in more detail by Alexandersson in [13], the company wanted to develop and test a new production layout in conjunction with a major expansion of the factory. Blue-collar workers were involved right from the start and they generated 32 partial suggestions for a new layout. These partial suggestions were subsequently synthesised by the facilitator in collaboration with the company into a number of overall proposals. After an elimination and selection process, four of these were presented to the blue-collar workers. They selected one of these proposals for simulation in KLF. This resulted in extremely high engagement and the one employee who was rather sceptical at first changed attitude completely once the simulation was underway. The feedback from the blue-collar workers during simulation was much more detailed and specific than was the case for the layout proposal when it was presented as a 2D paper-based mock-up, much similar to a layout drawing. In general, operators often have difficulties to oversee and grasp all consequences of a new production solution if this solution is presented as a layout drawing only [14, p 145]. Timon [15] also reports improved feedback from operators when they are asked to participate in modelling \& simulation activities, as compared to interviews and other ways of capturing operator knowledge.

A phase of particular interest was modelling the case company's production in KLF. The product that is normally used in KLF for training purposes is a chair from IKEA's play furniture (this is used as default, but companies can suggest to use other products). The case company manufactures high-end sun shields, and the blue-collar workers decided that since this product consists of two main parts (frame and cloth), it could be represented by a chair's side and a back rest respectively. In this case, the simulation task was not to produce chairs, but to make sure that the frame and the cloth belonging to a specific customer order arrived at the marriage station simultaneously. This is a good example of how this type of simulation can help to focus on main characteristics instead of putting effort into discussing details of limited relevance. In order to simulate a realistic case, an order book from a month with high demand (and much overtime) was selected for the simulation. Cycle times for the workstations during the simulation were based on realistic ratios estimated from real production. The simulation cycle times were short enough to play a large enough number of rounds, but long enough to allow reflection. In this particular case, two facilitators supported the modelling \& simulation process: one with good knowledge on the company's production and one with extensive experience from both LP and KLF.

Two alternating groups carried out the simulations, meaning that between simulation rounds, each employee changed role between that of participant and that of observer. It would have been possible to simulate in two different groups, but at least during LP training this often results in unwanted competition between teams. Simulating with one simulator instantiation and two alternating groups stimulated further reflection and discussion. The employees continued along this line after the simulation sessions; they generated some more layout improvement suggestions afterwards. This is similar to LP training, where the target is not to solve a specific 
problem, but rather to train problem-solving and opportunity-seeking capabilities, and to stimulate development of a new attitude. Likewise, in the case at hand, the purpose of the simulation is partly to solve a specific production problem (or exploit the opportunities presented by the factory floor expansion) and partly to initiate a culture change. An added bonus for the company was that not all floor space was needed for the new production layout. The new layout proved to make much more efficient use of the available space than initially thought, due to amongst others reduced total transport length and reduced need for buffers. This illustrates the potential benefits of involving blue-collar workers in production development processes at an early stage.

\subsection{A model for a participatory modelling \& simulation process}

The general process for engaging with a company in using an LP simulator for exploring innovative production solutions (as used in the example above) can be described by a model similar to the double diamond model as proposed by the Design Council [16] for product development. According to this model, from an initial problem/opportunity formulation as starting point, first a diverging process is used to explore the problem. The second phase in which the scope, limitations and project plan are defined, culminating into a so-called "design brief". This is the first diamond. The second diamond has the design brief as starting point and starts with a diverging phase where alternative solutions are being developed. This phase is followed by a converging phase that ends with the delivery of the solution. Figure 4 shows the first version of such a model for participatory modelling \& simulation for production innovation using LP simulators. Before the actual simulation, firstly the scope and objectives have to be defined, followed by a decision regarding which initial solutions to simulate and how. Thus, this first stage is a double diamond process in itself.

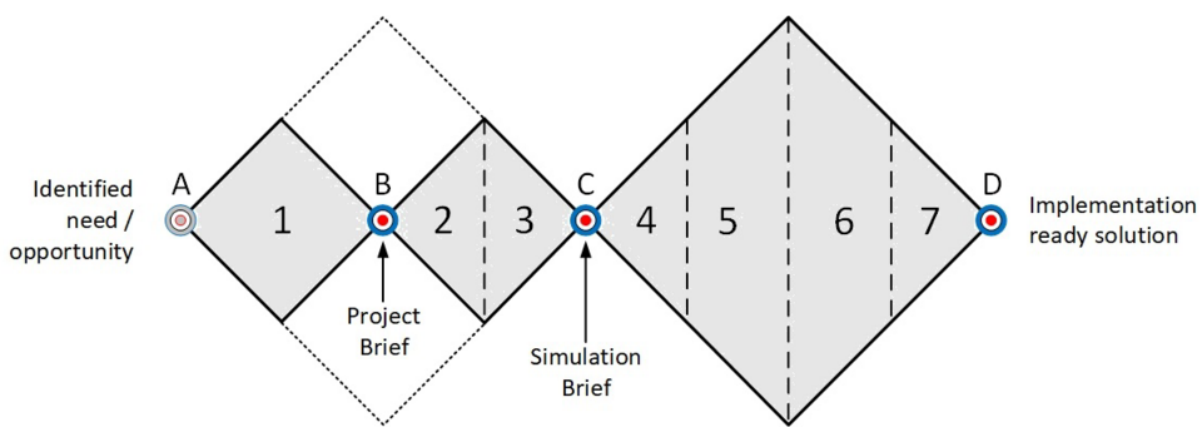

Fig. 4. Double diamond model for KLF participatory modelling \& simulation process

The model above shows four different main targets during the participatory modelling and simulation process for innovative production:

A: Initial state: Identified need/opportunity. At this stage, the overarching target (a desired future condition) is usually not well-defined.

B: Clarified target and level of ambition for the project. Aligned expectations and consensus on scope and delimitations of the simulation. 
C: Selected solution(s) for the participatory modelling \& simulation phase, target for simulation clarified and agreed upon by all stakeholders.

D: A new production layout/solution has been delivered and is ready for implementation phase at the company.

The model also shows that a typical participatory modelling \& simulation process for testing innovative production solutions in KLF can be divided into seven main phases. These are:

1. Clarification of task \& scope, alignment of expectations

2. Generation of partial solutions involving blue-collar workers

3. Synthesis of partial solutions into one or more overall solutions

4. Participatory modelling involving blue-collar workers

5. Exploration of alternatives through immersive simulation

6. Selection and refinement of alternative potential solutions using the simulator

7. Continued work at the company: Further improvements and detailed design of factory layout

As this is a general overview of typical phases, some variants of this general process may be appropriate in specific cases, as discussed below.

\subsection{Discussion and future work}

People in the three roles described above (process owner, facilitator, domain expert) will have shifting tasks and responsibilities in the different phases. The tasks and responsibilities can also vary somewhat from case to case. Such details can be decided upon in Phase 1. For instance, the facilitator usually has no tasks in Phase 7 other than possibly that of LP expert. This is quite similar to the role of the facilitator for continuous improvement cycles, see Figure 2, to the right.

In particular, the role of company management can be defined in Phase 1 . Generally speaking, it is important that management is engaged in the process, but the degree of activity may vary. In one case, the managing director of a small company participated in the simulations but deliberately took a relatively passive role during the improvement discussions so as not to steer these in a certain direction and to stimulate feedback and suggestions from shop floor operators. This playing down of hierarchy is mentioned as an important contributing success factor for LP by Bicheno in [17].

In many cases, a workshop on creative methods (such as brainstorming, 6-3-5 method [18], or negative idea generation) prior to Phase 2 can be helpful. This can lower the threshold for employees to suggest innovative non-traditional solutions. It also helps them to understand that most suggestions are valuable and that there is not a single "best solution". It is generally acknowledged that for many organisational problems, employees thinking together can generate alternative solutions each of which will be effective, as long as the selected solution is jointly agreed upon by these employees [8].

Between Phase 3 and Phase 4 in the model presented above, a workshop can be held to clarify the purpose of the simulation and to motivate participants through storytelling (good examples and showcases). This typically also is the border between onsite discussions and offsite simulation. It is highly recommended by the authors to carry out the simulations in Phase 4-6 offsite as this reduces the risk for distraction, as also suggested in [19]. The workshop, if held offsite, can also be an opportunity for the 
participants to familiarize themselves with the equipment prior to Phase 4 . Alternatively, a small set of workstations could be demonstrated onsite for the operators to get used to the stations" "look \& feel".

If Target $D$ (a solution that is ready for implementation) would be reached already after Phase 6, then the simulation project has probably missed the overarching goal of creating a culture of innovation. The aim of the simulation is not only to provide decision support (lack of decision support is one of the innovation barriers for SMEs) but also to create a culture of innovation. Thus, the aim is not to provide a "near turnkey solution" at the end of the simulation. It is also rather unlikely that a simulated solution is implemented "as is" without any further improvement, as a simulation model always is a simplification and abstraction of a part of the real world; and not an identical copy $[10,20]$.

Currently, the authors are preparing more industrial cases in which to apply and test this approach and to validate the model presented above as supporting the participatory modelling and simulation process. These cases will also show whether the results from the case described in [13] (namely, that participatory modelling and simulation stimulates production innovation in manufacturing SMEs) can be generalised.

The authors see monitoring of LP maturity as a means for an organisation to maintain development momentum and to stimulate internal discussion throughout the organisation. For instance, Tortorella et al. [21] suggest that service departments can benefit from co-evolving their LP maturity together with production departments. However, many maturity measurement tools are cumbersome to use for SMEs and often require consultancy-led activities, which makes them practically and economically unsuitable for use on a regular basis. Moreover, inclusion is not included as a maturity measure in tools reported on by Shah and Ward [22], and only indirectly in the tool proposed by Chiva et al. [23]. However, the latter tool is simpler than most other tools and would be more appropriate as a self-assessment tool for SMEs. Various authors suggest that for SMEs, relatively simple qualitative tools might be more appropriate than quantitative tools [24, 25]. This is supported by statements from industry, even larger manufacturing enterprises. The authors have developed a first version of a LP maturity self-assessment tool that is currently being tested in a pilot project within a food processing company.

\section{Conclusion}

Training effects from training in a simulated LP environment, and in particular training transfer to the workplace, can be stimulated when the training and continuous improvement activities are described by similar models. When using a simulator as innovation testbed, participatory modelling \& simulation facilitates capturing tacit knowledge. A case study confirms that participatory modelling \& simulation stimulates engagement as well as acceptance of results. Moreover, it creates a new culture of actively seeking potential improvements that is transferred into the workplace. A "double diamond" model describing such a process for simulator-assisted production innovation has been introduced and successfully applied in a case study. This model will be tested more and developed further in future work. Another topic for future work is the development of a tool for daily self-assessment of LP maturity in SMEs. 


\section{Acknowledgements.}

This work has been supported in part by funding from the European Regional Development Fund (Grant 20201681) and by funding from Region Värmland (Sweden).

\section{References}

[1] K. Luttik, Lean learning / learning lean at Scania, In: European Lean Educators Conference (ELEC), Nijmegen, NL, 2017.

[2] L.J. De Vin, L. Jacobsson. Karlstad Lean factory: An Instructional Factory for Game-based Lean manufacturing training, Production \& Manufacturing Research, 5:1, 2017, pp. 268-283.

[3] M. Koops, M. Hoevenaar. Conceptual Change During a Serious Game: Using a Lemniscate Model to Compare Strategies in a Physics Game, Simulation \& Gaming, 44(4), 2012. pp. 544-561

[4] R. Moen, 2009, Foundation and History of the PDSA Cycle. Accessed: 15.05.2019. [Online]. Available: https://deming.org/uploads/paper/PDSA_History_Ron_Moen.pdf.

[5] Wilson Perumal. 2013, Implementing the 7 Element OEMS: Plan-Do-Check-Adjust. Accessed: 07.03.2019. [Online]. Available: http://www.wilsonperumal.co,m/blog.

[6] N. Radziwill, 2011, PDCA vs. PDSA: What's the Difference? Accessed: 14.09.2019. [Online]. Available: https://qualityandinnovation.com/2011/08/26/

[7] D. Wood, J.S. Bruner, G. Ross. The Role of Tutoring in Problem Solving, J. Child Psychol. Psychiat., Vol 17, 1976, pp. 89-100.

[8] N.M. Dixon. The Hallways of Learning. Organizational Dynamics, Spring 1997, pp. 23-34.

[9] L.J. De Vin, L Jacobsson, J. Odhe. Simulator-assisted lean production training. Production \& Manufacturing Research, 7:1, 2019, pp. 433-447.

[10] A. Rosenblueth, N. Wiener. The role of models in science. Philosophy of Science, 12(4), 1945, pp. 316321.

[11] B. Aures. Game Design for a Lean (Food) Simulation at AUDI AG, In: European Lean Educators Conference (ELEC). Nijmegen, NL, 2017.

[12] J. Stirna, A. Persson, K. Sandkuhl. Participative enterprise modeling: Experiences and recommendations, Lecture Notes in Computer Science, 4495, 2017, pp. 546-560.

[13] J. Alexandersson. Change of layout and production flow, Student Thesis Karlstad University, 2018. [in Swedish].

[14] M. Bellgran, K. Säfsten. Produktionsutveckling - utveckling och drift av produktionssystem, Studentlitteratur, Lund, 2005 [in Swedish].

[15] F. Timon. Round Table Discussion Session, In: 2nd IWSMSL, Dublin, 2005.

[16] Design Council, 2015, Accessed 24.05.2019. [Online]. Available: http://www.designcouncil.org.uk/.

[17] J. Bicheno. Lean: the forgotten, the ignored, the possible, In: European Lean Educators Conference (ELEC). Nijmegen, NL. 2017.

[18] D. Ullman, The Mechanical Design Process, McGraw-Hill, New York, 2010.

[19] M. Tawalbeh, R. Riedel, M. Dempsey, C. Emanuel. Lego ${ }^{\circledR}$ Serious Play ${ }^{\circledR}$ as a Business Innovation enabler, In: European Lean Educators Conference (ELEC), Braga PT, 2018, pp. 90-98.

[20] L.J. De Vin, Simulation, Models, and Results: Reflections on their Nature and Credibility, In: FAIM2015, Middlesborough, UK, 2015, Vol II, pp. 148-155.

[21] G.L. Tortorella, D. Fetterman, P.A. Cauchick Miguel and R. Sawney, Learning organisation and lean production: an empirical research on their relationship, Int. Jrnl. of Production Research, 2019. https://doi.org/10.1080/00207543.2019.1633028.

[22] R. Shah, P.T. Ward. Defining and developing measures of lean production, Journal of Operations Management, 25, 2007, pp. 785-805.

[23] R. Chiva, J. Alegre, R. Lapiedra. Measuring Organisational Learning Capability among the Workforce. International Journal of Manpower, 28-3/4, 2007, pp. 224-242.

[24] V. Chiesa, P. Coughlan, and C. A. Voss, "Development of a technical innovation audit," Journal of Product Innovation Management, vol. 13, no. 2, 1996, pp. 105-136.

[25] A. Goienetxea Uriarte, M. Urenda Moris, M. Jägstam, Lean, simulation and optimization: A maturity model, In: 2017 IEEE Int. Conference on Industrial Engineering and Engineering Management, Singapore, 2017, pp. 1310-1315. 